MODELING, IDENTIFICATION AND CONTROL, 2004, vOL. 25, No. 4, 223-235

doi:10.4173/mic.2004.4.2

\title{
DVL Velocity Aiding in the HUGIN 1000 Integrated Inertial Navigation System
}

\author{
BJØRN JALVING*, KENNETH GADE*, KRISTIAN SVARTVEIT*, \\ ARE WILLUMSEN $\dagger$ and ROBERT SØRHAGEN $\ddagger$
}

Keywords: Autonomous underwater vehicle, aided inertial navigation, Kalman filter, Inertial measurement unit, Doppler velocity log

\begin{abstract}
The RDI WHN-600 Doppler Velocity Log (DVL) is a key navigation sensor for the HUGIN 1000 Autonomous Underwater Vehicle (AUV). HUGIN 1000 is designed for autonomous submerged operation for long periods of time. This is facilitated by a low drift velocity aided Inertial Navigation System (INS). Major factors determining the position error growth are the IMU and DVL error characteristics and the mission plan pattern. For instance, low frequency DVL errors cause an approximately linear drift in a straight-line trajectory, while these errors tend to be cancelled out by a lawn mower pattern. The paper focuses on the accuracy offered by the DVL. HUGIN 1000 is a permanent organic mine countermeasure (MCM) capacity on the Royal Norwegian Navy MCM vessel KNM Karmøy. HUGIN 1000 will be part of the NATO force MCMFORNORTH in fall 2004.
\end{abstract}

\section{Introduction}

Kongsberg Maritime and FFI have cooperated in developing the HUGIN family of autonomous underwater vehicles. HUGIN 3000 was the world's first AUV used in commercial survey operations (Chance et al., 200; George et al., 2003; Vestgård et al., 2001). The three HUGIN 3000 class AUVs have been used in areas as diverse as the Gulf of Mexico, the Mediterranean, Brazil, West Africa, the North Sea and the Norwegian Sea. Building on more than 5 years of field experience with commercial AUV use, the HUGIN 1000 vehicle was developed, targeting the military market and civilian research, mapping and monitoring applications. Compared to HUGIN 3000, HUGIN 1000 is smaller, easier to handle, has lower depth rating and shorter endurance, but software, electronics and system design are almost identical (Hagen et al., 2003). The first HUGIN 1000 was delivered to the Royal Norwegian Navy in January 2004. The vehicle is permanently installed on the KNM Karmøy mine countermeasures (MCM) vessel. Organic AUV MCM operations as a concept is continuously developed, refined and evaluated while HUGIN 1000 is contributing to military worth in military exercises and operations. In fall 2004 KNM Karmøy and HUGIN 1000 will be part of the standing NATO force MCMFORNORTH.

*Norwegian Defence Research Establishment (FFI), P.O. Box 25, 2027 Kjeller, Norway, emails: bjorn.jalving@ffi.no, kenneth.gade@ffi.no, kristian.svartveit@ffi.no

†UniK - University Graduate Center, P.O. Box 70, NO-2027 Kjeller, Norway, email: are.b.willumsen@unik.no

$\Varangle$ Kongsberg Maritime, P.O. Box 111, NO-3191 Horten, Norway, email: robert.sorhagen@ kongsberg.com

(C) 2004 RD lnstruments. Reprinted from Proceedings from ADCPs in Action, Nice, June 3-4, 2004 

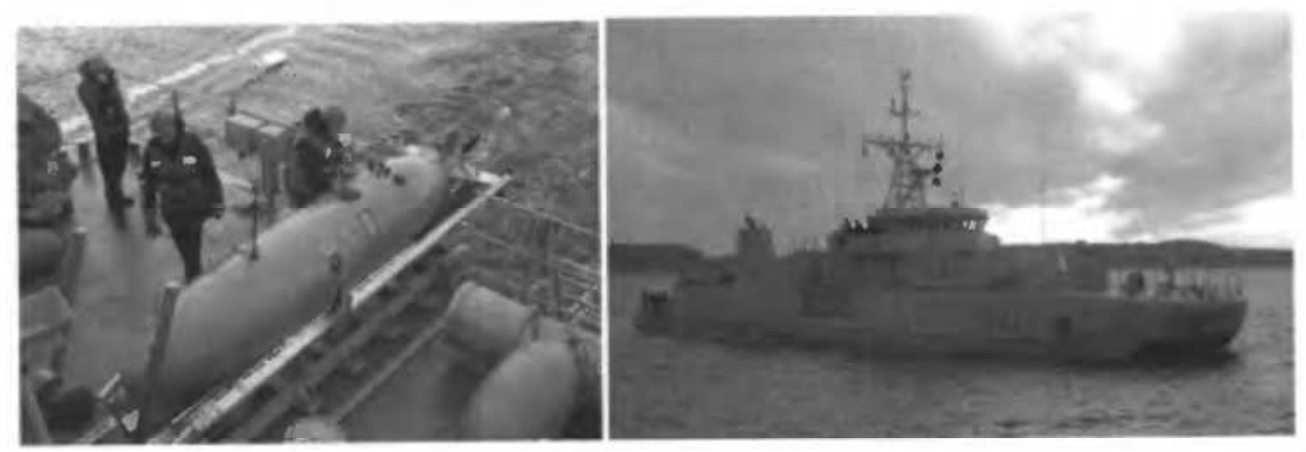

Figure 1. Left: HUGIN 1000 on aft deck of KNM Karmøy. Right: KNM Karmøy mine countermeasures vessel.

Autonomous operation in deep water or covert military operations requires the AUV to handle submerged operation for long periods of time. The state of the art solution embedded in the HUGIN AUVs is a Doppler Velocity Log (DVL) aided Inertial Navigation System (INS) that can integrate various forms of position measurement updates. The HUGIN vehicles are using the RDI WHN-300 and WHN-600 DVLs. This paper presents the HUGIN integrated inertial navigation system in general and discusses DVL velocity aiding in particular. The data examples are taken from the new HUGIN 1000, still undergoing operational evaluation.

\section{HUGIN 1000 Navigation System Philosophy}

\subsection{Integrated Inertial Navigation System Structure}

In Figure 2 the structure of the HUGIN integrated inertial navigation system and a summary of the HUGIN navigation toolbox is shown. The Inertial Navigation System (INS) calculates position, velocity and attitude using high frequency data from an Inertial Measurement Unit (IMU). An IMU consists of three accelerometers measuring specific force and three gyros measuring angular rate, relative to inertial space. An extended Kalman filter will, in a mathematically optimal manner, utilize a wide variety of navigation sensors for aiding the INS. The Kalman filter is based on an error-state model and provides a much higher total navigation performance than would be obtained from the independent navigation sensors.

A core low drift DVL aided inertial navigation system is capable of handling submerged autonomous operation for long periods of time. In Figure 2, the core DVL aided INS system consists of the IMU and the navigation equations, the error state Kalman filter and the following aiding sensors: DVL, compass (optional) and pressure sensor.

Depending on position accuracy requirements, the navigation system must get occasional position measurement updates. GPS surface fixes is the preferred method for position updates when moderate water depths, mission efficiency and covertness requirements allow. For submerged position updates, the HUGIN vehicles come with bathymetric terrain navigation and acoustic ranging to underwater transponders (UTP). If HUGIN is in acoustic vicinity of its mother ship, DPGS-USBL (ultra short baseline) aiding can be used. NavLab post-processing allow for maximum position accuracy in demanding applications (Gade, 2003). SAS velocity aiding is a 


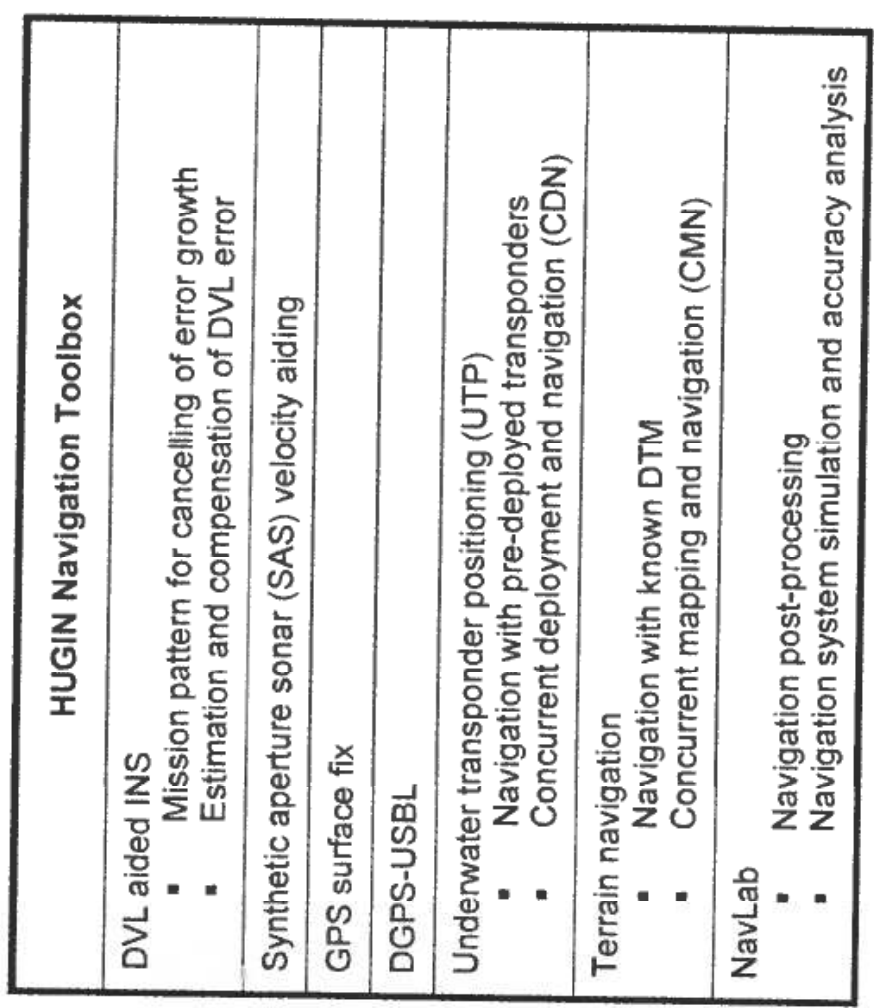

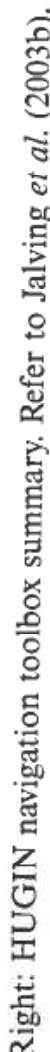

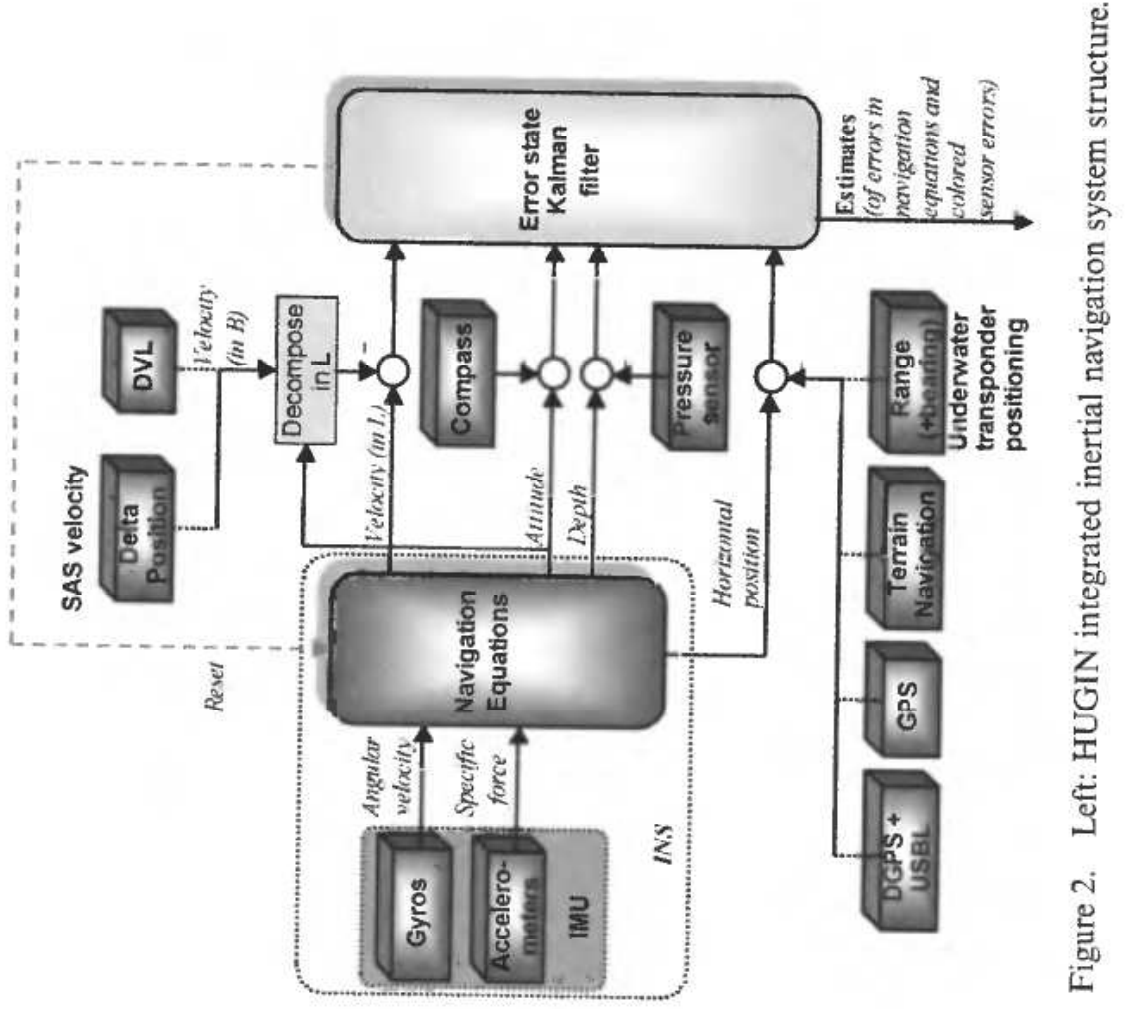


Table 1. INS classes. RLG-Ring Laser Gyro, FOG-Fiber Optic Gyro

\begin{tabular}{lccc}
\hline Class & Gyro technology & Gyro bias & Accelerometer bias \\
\hline$>10 \mathrm{nmi} / \mathrm{h}$ & RLG, FOG & $1 \% \mathrm{~h}$ & $1 \mathrm{milli} \mathrm{g}$ \\
$1 \mathrm{nmi} / \mathrm{h}$ & RLG, FOG & $0.005 \% \mathrm{~h}$ & 30 micro g \\
\hline
\end{tabular}

potentially rewarding method, however, not yet demonstrated on real AUV data. More information on the toolbox and use of the toolbox in different applications can be found in Jalving et al. (2003b).

Inertial navigation systems are usually classified by the standard deviation of the positional error growth of their free inertial (unaided) performance (see Table 1). A free unaided INS will, after a short period of time, have unacceptable position errors. The HUGIN navigation system can interface any IMU, but for most applications the IMU will be in the navigation grade $(1 \mathrm{nmi} / \mathrm{h})$ class.

\section{DVL Velocity Aiding}

\subsection{DVL Alternatives}

DVL accuracy is dependent on frequency. Higher frequency yields better accuracy at the sacrifice of decreased range as illustrated in Table 2. Prioritization between range and accuracy is dependent on the application. RDI WHN-300 is standard outfit for the HUGIN 3000 AUVs that operate in waters down to $3000 \mathrm{~m}$ and is typically followed by a mother ship equipped with DGPS and USBL for continuous position updates. HUGIN 1000 on the other hand, normally operates in autonomous mode. Prioritizing autonomous submerged navigation accuracy, standard HUGIN 1000 is equipped with RDI WHN-600.

\subsection{Simplified Error Analysis Straight Trajectories}

The simplified error analysis presented in this section is useful for understanding the basic mechanisms of a DVL aided INS and assessing how IMU and DVL sensor accuracy is determining the overall position accuracy.

The horizontal position drift in a DVL-aided INS is determined by the error in the estimated Earth-fixed velocity (i.e. North and East velocity). The main contributors to this error are:

Table 2. RDJ Workhorse Navigator Doppler Velocity Log accuracy and range specifications, RD Instruments (2003). o.s. - of speed. The accuracy figures can be interpreted as $1 \sigma$ values. The error consists of two independent varying components; scale factor error and bias. The total error is the root mean square (rms) of the two components.

\begin{tabular}{ccc}
\hline Frequency & Long term accuracy & Range \\
\hline $150 \mathrm{kHz}$ & $\pm 0.5 \%$ o.s. $\pm 2 \mathrm{~mm} / \mathrm{s}$ & $425-500 \mathrm{~m}$ \\
$300 \mathrm{kHz}$ & $\pm 0.4 \%$ o.s. $\pm 2 \mathrm{~mm} / \mathrm{s}$ & $200 \mathrm{~m}$ \\
$600 \mathrm{kHz}$ & $\pm 0.2 \%$ o.s. $\pm 1 \mathrm{~mm} / \mathrm{s}$ & $90 \mathrm{~m}$ \\
$1200 \mathrm{kHz}$ & $\pm 0.2 \%$ o.s. $\pm 1 \mathrm{~mm} / \mathrm{s}$ & $30 \mathrm{~m}$ \\
\hline
\end{tabular}


- Error in the body-fixed velocity

- Error in heading.

The error in estimated body fixed velocity is mainly determined by the low-frequency error in the DVL itself (without position aiding this error is not observable when going at a straight line). High frequency velocity errors are estimated by means of the accelerometers. Even the most accurate INS will without aiding after a short period of time have a velocity uncertainty larger than the DVL accuracy. Referring to Table 2, a $300 \mathrm{kHz}$ DVL typically have a scale factor type of error of $0.4 \%$ of speed, contributing to an along track error drift of $0.4 \%$ of traveled distance, or 28.8 $\mathrm{m} /$ hour for an AUV traveling at $2 \mathrm{~m} / \mathrm{s}$ ( 4 knots). However, there are ways to improve the DVL accuracy: Sacrificing range, the $600 \mathrm{kHz}$ and $1200 \mathrm{kHz}$ versions have an accuracy specification of $0.2 \%$ of speed, corresponding to $0.2 \%$ of traveled distance, or $14.4 \mathrm{~m} /$ hour (AUV speed $2 \mathrm{~m} / \mathrm{s}$ ). The scale factor error is observable by the Kalman filter when position measurements are available or when the AUV is turning. Thus, the Kalman filter can compensate for part of the scale factor error when running more complex missions than a straight line. This is discussed in Section 3.3.

The error in heading is determined by the gyrocompassing capability of the integrated system. The heading estimation error will typically be of low frequency, corresponding to non-observable gyro bias dynamics. Referring to Table 1 , a $1 \mathrm{nmi} /$ h navigation class IMU typically gyrocompass to an accuracy of $\sigma(\delta \psi)=0.02^{\circ}$ I $\cos$ (latitude). This corresponds to an across track error drift of $\sigma(\delta \psi) \cdot 100 \%$ of traveled distance $\left(\sigma(\delta \psi)\right.$ in radians). At $45^{\circ}$ latitude, this equals $0.05 \%$ of traveled distance, or $3.4 \mathrm{~m} /$ hour at $2 \mathrm{~m} / \mathrm{s}$ AUV speed. DVL error in AUV body y-direction also contributes to across track drift. If there is no current, the scale factor error can be ignored leaving the constant error defined in Table 2. The WHN-600 accuracy specification of $1 \mathrm{~mm} / \mathrm{s}$ contributes with $3.6 \mathrm{~m} /$ hour $1 \sigma$. Assuming uncorrelated error processes, across track error drift amounts to $5.0 \mathrm{~m} /$ hour at $2 \mathrm{~m} / \mathrm{s}$ AUV speed.

In Jalving et al. (2003a) position accuracy for an INS with $1 \mathrm{nmi} / \mathrm{h}$ IMU and $1200 \mathrm{kHz}$ DVL following a straight line was simulated. Along track position error drift was in the order of $8 \mathrm{~m} /$ hour while cross track position error drift was in the order of $2.5 \mathrm{~m} /$ hour. This is a somewhat smaller drift than predicted by the simplified error analysis. There are two main reasons for this: the Kalman filter compensates for a scale factor error estimated when position measurements were available and the actual scale factor error is modeled as a first order Markov process and not a constant error. Choosing time constants that realistically reflect the physical error processes is very important when estimating DVL aided INS error drift and when tuning the Kalman filter for real applications. This explains the importance of characterization of the DVL errors discussed in Section 5.1.

\subsection{Countering DVL Aided INS Position Error Growth}

For a submerged AUV without position updates, the position error growth of a DVL aided INS can be countered by:

1. Mission pattern for canceling of error growth

2. Kalman filter estimation and compensation of DVL error

The accuracy estimates in Section 3.2 are valid for straight-line trajectories. Since the main error contributors of DVL aided INS is body fixed velocity and heading, a canceling effect of the error growth is obtained when for instance running a lawn 
Table 3. Typical reduction in position error drift for a DVL aided INS when comparing a straight-line trajectory with a lawn mower pattern, RD Instruments (2003). The numbers apply for a $1200 \mathrm{kHz}$ DVL and a $1 \mathrm{nmi} / \mathrm{h}$ IMU at 45 latitude.

\begin{tabular}{lcc}
\hline $\begin{array}{l}\text { Position error drift } \\
\text { (\% of traveled distance) }\end{array}$ & Straight line & $\begin{array}{c}\text { Lawn mower pattern } \\
\text { with } 1 \mathrm{~km} \text { lines }\end{array}$ \\
\hline Along track & $0.11 \%$ & $0.01 \%$ \\
Across track & $0.03 \%$ & $0.001 \%$ \\
\hline
\end{tabular}

mower pattern. The canceling effect increases with the stability of the body fixed velocity error and heading error. Also the canceling effect increases with shorter line lengths.

A second important effect of maneuvering is that the velocity error actually becomes observable by means of comparing expected centripetal acceleration with measured acceleration from the IMU. If the velocity error is the same during the maneuver (i.e. when it is observed) as it is in the following line, the error is estimated and compensated for. The error growth when running long straight lines can thus be reduced by adding $360^{\circ}$ turns at regular intervals. However this mechanism requires accurate DVL time stamping and unchanged DVL error characteristics in the turn (refer to Sections 5.3 and 5.4), and is thus challenging to achieve on real data in real-time.

The two effects combined are very effective, as seen in Table 3, which contains results from NavLab simulations (see Gade (2003) and Jalving et al. (2003b) for NavLab description).

\section{Heading Estimation Accuracy and Importance of DVL Mounting Accuracy}

Gyrocompassing (heading estimation by observing Earth rotation) is an inherent part of an optimal estimator as long as it gets position or velocity measurement updates. The IMU gyro bias limits the accuracy of the INS heading estimate. Ignoring gyro angular random walk and acceleration uncertainty, INS gyro compassing accuracy is approximately given by

$$
\sigma\left(\delta \psi_{I M U}\right)=\frac{\Delta \omega_{\text {gyrobias }}}{\omega_{I E} \cos \mu}
$$

where $\sigma\left(\delta \psi_{I M U}\right)$ is heading accuracy due to IMU gyro bias, $\Delta \omega_{\text {gyrobias }}$ is gyro bias and $\omega_{I E} \cos \mu$ is the horizontal component of Earth's rotation rate ( $\mu$ is latitude).

The DVL measures the velocity vector in AUV body coordinates. The INS heading, pitch and roll estimates are used to transform this velocity into an Earth fixed coordinate system. If position measurements are available, the Kalman filter estimates errors in velocity and heading. Ignoring white measurement noise, this mechanism contributes with a heading accuracy of

$$
\sigma\left(\delta \psi_{D V L}\right)=\frac{\Delta v_{D V \text { Lerror, } y}}{v_{E B, x}^{B}}
$$

where $\sigma\left(\delta \psi_{D V L}\right)$ is heading accuracy due to DVL, $\Delta v_{D V L e r r o r, y}$ is low frequent DVL error in AUV body $y$ and $v_{E B, x}^{B}$ is AUV forward velocity.

Insertion of typical figures for a navigation grade IMU and a RDI WHN-600 
results in $\sigma\left(\delta \psi_{I M V}\right)=0.05^{\circ}$ at $45^{\circ}$ latitude and $\sigma\left(\delta \psi_{D V L}\right)=0.1^{\circ}$. In theory, these mechanisms will work in concert improving the overall heading accuracy. However, if there is a mounting misalignment between the IMU and the DVL, which is not accounted for in the Kalman filter, they can actually be counter productive. Thus, DVL mounting accuracy is very important to achieve the accuracy offered by the IMU and the DVL sensors themselves.

In HUGIN 1000, special care is taken during production to mount all sensors, including IMU and DVL, as accurately as possible. After assembling, NavLab is used to estimate any DVL misalignment. The IMU and DVL are mounted with steering pins. Thus, if the sensors are dismounted for service or inspection, the steering pins will assure the same orientation when remounted.

\section{DVL Error Analysis}

\subsection{DVL Error Budget}

As discussed in Section 3.2 the magnitude and the frequency characteristics of the DVL error is important to the position drift in the integrated inertial navigation system. White measurement noise causes a position uncertainty that increases with square root of time. A constant error causes a linear position error drift, which potentially can be cancelled out by a lawn mower pattern. From a Kalman filter point of view, the physical error process should be modeled truthfully to get as accurate position estimate as possible.

Table 4 summarizes a scale factor error budget for a $300 \mathrm{kHz}$ broadband DVL (Lester, 2001). The two constant components are candidates for estimation and compensation. However, the major part of the error budget is time varying. This is in accordance with Kalman filter estimates in HUGIN 1000, see Figure 6. Further investigation, identification and understanding of the DVL error processes are of priority because of the prospects of improving the overall navigation accuracy by more accurate error modeling.

Table 4. $300 \mathrm{kHz}$ DVL scale factor error budget. Copied from Lester (2001)

\begin{tabular}{lcc} 
Error source & $\begin{array}{c}\text { Scale factor } \\
\text { error (\%) }\end{array}$ & Time varying \\
\hline $\begin{array}{l}\text { Absorption bias. } \\
2 \text { sigma over } 0 \text { to } 80 \% \text { of range. }\end{array}$ & 0.081 & Yes \\
$\begin{array}{l}\text { Terrain bias. } \\
2 \text { sigma about centre of typical range of backscatter slopes. }\end{array}$ & 0.144 & Yes \\
$\begin{array}{l}\text { Sound speed temperature dependence. } \\
\text { Assuming } 0.5^{\circ} \text { rms uncertainty. }\end{array}$ & 0.100 & Yes \\
$\begin{array}{l}\text { Sound speed salinity dependence. } \\
\text { Assuming } 0.5 \text { ppt rms uncertainty. }\end{array}$ & 0.040 & Yes \\
$\begin{array}{l}\text { Sidelobes } \\
\text { (4 dB rms) }\end{array}$ & 0.040 & Yes \\
$\begin{array}{l}\text { Beam angle } \\
\text { Other minor sources (clock drift, etc.) }\end{array}$ & 0.086 & No \\
Total' (rms of values above) & 0.080 & No \\
\hline
\end{tabular}




\subsection{Importance of Sound Speed Accuracy}

The velocity scale factor is proportional to the sound speed at the transducer. The WHN DVLs can be set to compute sound speed based on internal sensors or sent data of salinity, temperature and depth. If there is an error, correct sound speed can be post-processed using the following equation (see RD Instruments, 1996):

$$
v_{\text {corrected }}=v_{\text {uncorrected }} \frac{C_{\text {real }}}{C_{A D C P}}
$$

where $C_{\text {real }}$ is the true sound speed at the transducer and $C_{A D C P}$ is the sound speed recorded by the ADCP. Using this formula the DVL error due to wrong sound velocity estimate can be computed as:

$$
\delta v_{D V L}=\frac{v_{\text {uncorrected }}}{C_{A D C P}} \delta C
$$

where $\delta v_{D V L}$ is DVL error and $\delta C$ is sound velocity error. Assuming $C_{A D C P}=1500 \mathrm{~m} /$ $\mathrm{s}$ and $v_{\text {uncorrected }}=2 \mathrm{~m} / \mathrm{s}$, a sound velocity error, $\delta C=3 \mathrm{~m} / \mathrm{s}$, causes a DVL error of $0.2 \%$ of speed. The sound speed induced velocity error should be considerably less than the DVL scale factor error (refer to Table 2). A sound speed accuracy of $0.5 \mathrm{~m} / \mathrm{s}$ is a reasonable specification.

In the sea trial referred to in Section 6, the DVL calculated sound speed using the default value for salinity ( 35 parts per thousand), its internal temperature sensor and depth set by the HUGIN Control Processor. In Figure 3, CTD temperature and sound velocity calculation are compared with DVL temperature and sound velocity calculation. HUGIN 1000 is equipped with the oceanographic grade Falmouth
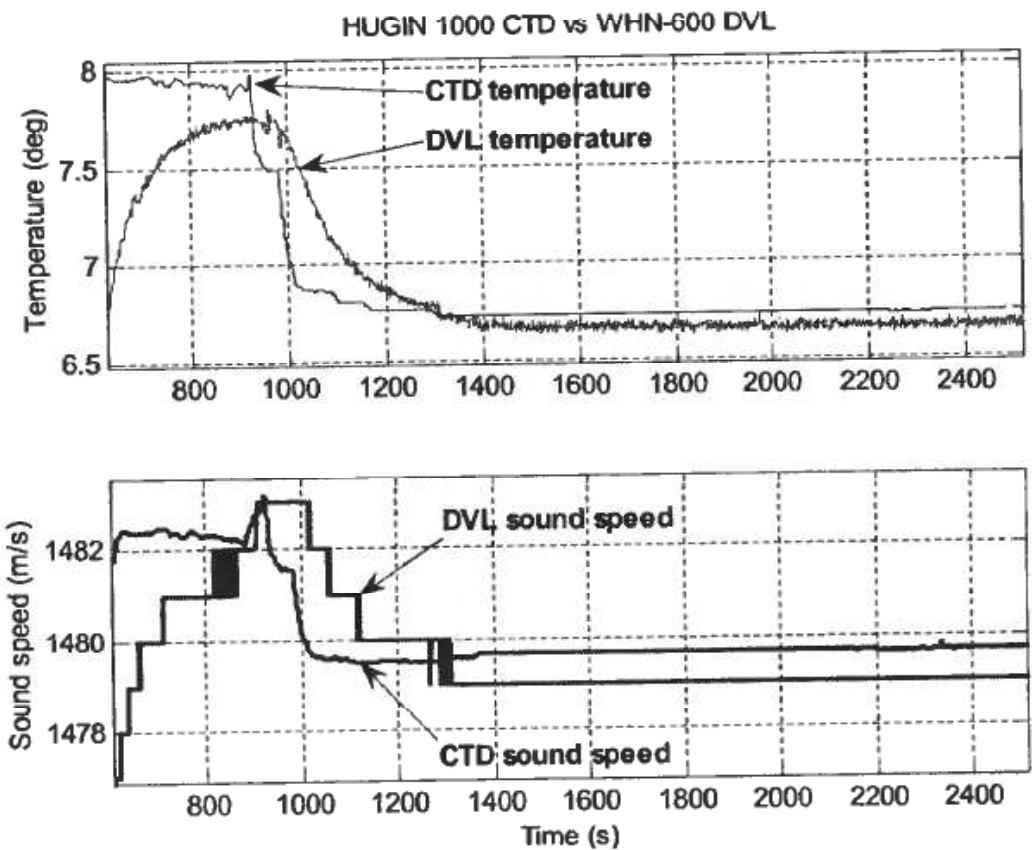

Figure 3. Comparison of temperature and sound speed between the WHN-600 (slowest reponse) and the HUGIN 1000 CTD sensor (fastest response). Note that least significant digit for sound speed in DVL datagram is $1 \mathrm{~m} / \mathrm{s}$. 
Scientific 2" Micro CTD (Falmouth Scientific, 2004). The DVL temperature sensor has a slower response time than the CTD temperature sensor, which affects the sound speed calculation. When stabilized, the difference between CTD sound speed and DVL sound speed is less than $1 \mathrm{~m} / \mathrm{s}$. AUVs experiencing rapid changes in temperature, typically due to depth changes, should preferably send a CTD computed sound speed to the DVL.

\subsection{Time Stamp Accuracy Requirements for DVL Aiding}

At $2 \mathrm{~m} / \mathrm{s}$ WHN-600 has a velocity accuracy of $0.004 \mathrm{~m} / \mathrm{s}$. In practice an underwater vehicle experience some dynamics. An ROV due to cable effects, an AUV during turns and when surfacing for GPS fixes. Thus IMU time stamp accuracy, DVL time stamp accuracy and DVL lever arm compensation are important to utilize the velocity accuracy offered by the DVL sensor itself.

Time stamp sensitivity to vehicle acceleration If the vehicle is accelerating, the velocity error, $\delta v$, due to incorrect time stamps when comparing DVL measurement with INS velocity in the Kalman filter, is given by:

$$
\delta v=a \cdot \delta t
$$

where $a$ is vehicle acceleration and $\delta t$ is time stamp error.

Time stamp sensitivity to vehicle rotation rate change If the AUV is rotating and there is an arm between the IMU and the DVL, the DVL velocity must be lever arm compensated before being compared to the INS velocity in the INS body frame.

Only considering one axis (for simplicity) DVL lever arm compensation, $v_{\text {comp }}$, is given by:

$$
v_{\text {comp }}=l \cdot \omega
$$

where $l$ is lever arm and $\omega$ is angular rotation rate $\left(\left|\omega_{E B}^{B}\right|\right)$. Error in DVL lever arm compensation, $\delta v_{\text {comp }}$, due to time stamp error is given by:

$$
\delta v_{\text {comp }}=l \cdot \dot{\omega} \cdot \delta t
$$

where $\dot{\omega}$ is change in angular rate and $\delta t$ is time stamp error. The error is proportional to the length of the lever arm. Preferably, lever arms should by vehicle design be as small as possible.

Time stamp requirements An obvious requirement is that time stamp induced velocity error due to vehicle acceleration and vehicle rotation rate change is less than DVL scale factor error. Analysis of typical HUGIN 1000 dynamics during GPS surface fixes, show that DVL time stamp accuracy should be better than $10 \mathrm{~ms}$. Such accuracy can be achieved by running the WHN-600 in external sync, time stamp the sync pulse and calculate correct time stamp from this value. This is a rather cumbersome method. Preferably the DVL output datagrams should include a DVL latency defined as midpulse-on-bottom to first character of output transmission.

\subsection{Geometric Effect of Turn Rate on DVL Accuracy}

In order to examine the geometric effect of turning the following assumptions are made: 
1. The vehicle has no heave, roll or pitch motion.

2. The four DVL beams are (from a horizontal point of view) directed $90^{\circ}$ to each other.

The DVL is rotated such that the first beam is an angle $\alpha$ (usually $45^{\circ}$ ) away from the forward direction. As there are no heave, roll or pitch motion, all Doppler effects come from sway, surge and yaw. Hence, only the horizontal part is considered. The vehicle's speed in each of the four DVL directions at transmission time can in terms of forward speed $v_{E B, x}^{B}$ and starboard speed $v_{E B, y}^{B}$, be expressed as

$$
\mathbf{v}_{D, \text { trans }}=\left[\begin{array}{l}
v_{D 1, \text { truns }} \\
v_{D 2, \text { trans }} \\
v_{D 3, \text { trans }} \\
v_{D 4, \text { trans }}
\end{array}\right]=\left[\begin{array}{rr}
\cos \alpha & \sin \alpha \\
-\sin \alpha & \cos \alpha \\
-\cos \alpha & -\sin \alpha \\
\sin \alpha & -\cos \alpha
\end{array}\right]\left[\begin{array}{l}
v_{E B, x}^{B} \\
v_{E B, y}^{B}
\end{array}\right]=\mathbf{A}(\alpha)\left[\begin{array}{l}
v_{E B, x}^{B} \\
v_{E B, y}^{B}
\end{array}\right]=\mathbf{A}_{\text {trans }}\left[\begin{array}{l}
v_{E B, x}^{B} \\
v_{E B, y}^{B}
\end{array}\right]
$$

Studying the case when the vehicle is turning, the vehicle has rotated an angle $\Delta \psi$, when the reflected beams return. The speed at reception in the four DVL directions is given by

$$
\mathbf{v}_{D, r e c}=\mathbf{A}(\alpha-\Delta \psi)\left[\begin{array}{c}
v_{E B, x}^{B} \\
v_{E B, y}^{B}
\end{array}\right]=\mathbf{A}_{r e c}\left[\begin{array}{c}
v_{E B, x}^{B} \\
v_{E B, y}^{B}
\end{array}\right]
$$

The relative Doppler shifts can, since $\mathbf{v}_{\text {trans }} \ll c$, be expressed as

$$
\Delta \mathbf{f} \approx \frac{\mathbf{v}_{D, \text { trans }}+\mathbf{v}_{D, \text { rec }}}{c}=\frac{1}{c}\left(\mathbf{A}_{\text {trans }}+\mathbf{A}_{r e c}\right)\left[\begin{array}{c}
v_{E B, x}^{B} \\
v_{E B, y}^{B}
\end{array}\right]
$$

The DVL measurement is given by

$$
\left[\begin{array}{c}
\tilde{v}_{E B, x}^{B} \\
\tilde{v}_{E B, y}^{B}
\end{array}\right]=\frac{c}{2}\left(\mathbf { A } _ { \text { trans } } ^ { T } ( \mathbf { A } _ { \text { trans } } ) ^ { - 1 } \left(\mathbf{A}_{\text {trans }}^{T} \Delta \mathbf{f}=\frac{1}{2}\left(\mathbf{I}+\left(\mathbf{A}_{\text {trans }}^{T} \mathbf{A}_{\text {trans }}\right)^{-1} \mathbf{A}_{\text {trans }}^{T} \mathbf{A}_{r e c}\left[\begin{array}{c}
v_{E B, x}^{B} \\
v_{E B, y}^{B}
\end{array}\right]\right.\right.\right.
$$

This yields a scale factor type of error. It is though negligible for most AUV missions because the angle $\Delta \psi$ is small. For instance with $50 \mathrm{~m}$ AUV altitude and $\dot{\psi}=6 \% \mathrm{~s}$ turn rate, results in $\Delta \psi \approx 0.5^{\circ}$.

\section{Results with RDI WHN-600}

The results shown in this section are based on a HUGIN 1000 test run in Horten (2004). HUGIN 1000 ran with DVL aiding only, but a surface ship followed HUGIN 1000 and made independent DGPS-USBL (ultra short baseline) measurements of the AUV position. These position measurements were later used in a NavLab postprocessing to provide an accurate position reference, with which the DVL aided INS results were compared. Please note that navigation systems are complex statistical processes and a number of separate missions should be run and compared for proper statistical performance characterization.

Figure 4 shows the $5.5 \mathrm{~km}$ straight-line trajectory run by HUGIN 1000. Prior to this line, the navigation system was aligned with position measurements. With DVL 


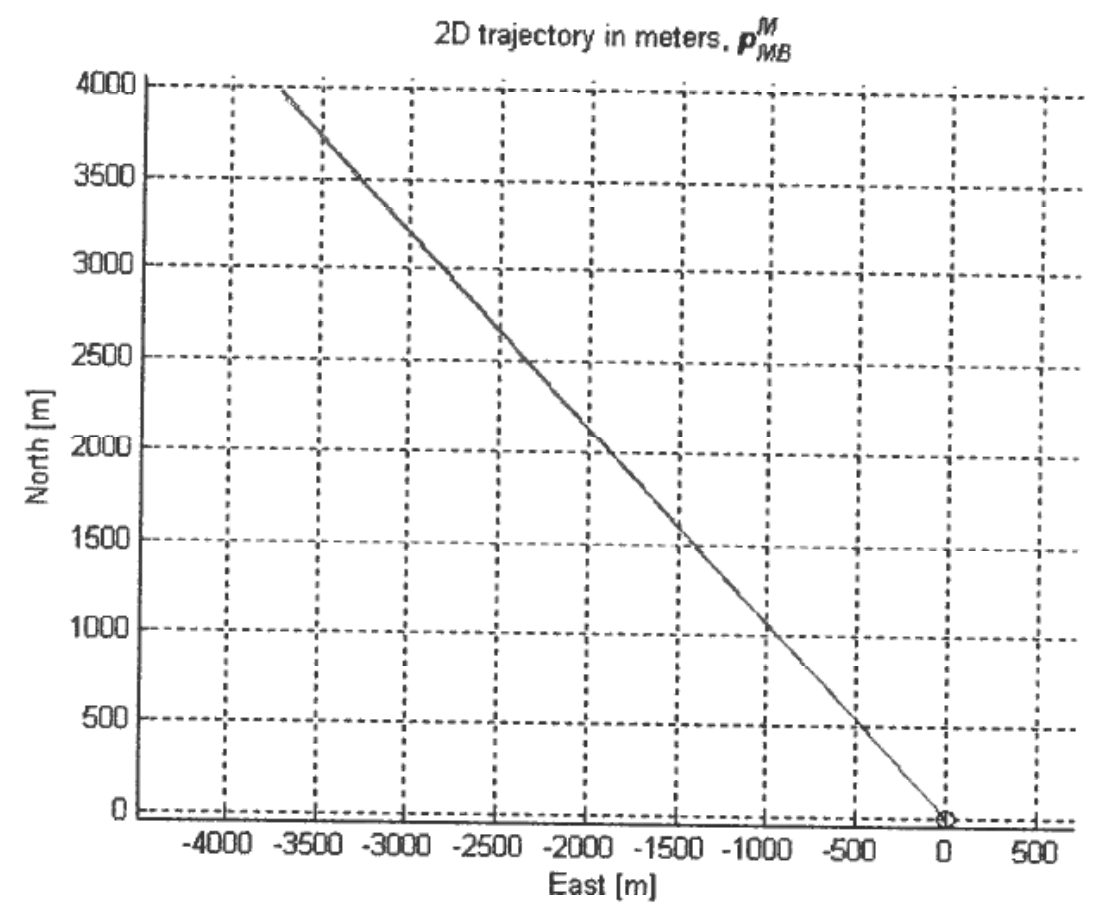

Figure 4. 2D trajectory. Black circle: Starting point. Black line: Post-processed position reference. Grey line: Estimated (Kalman filtered) position using only DVL aiding.
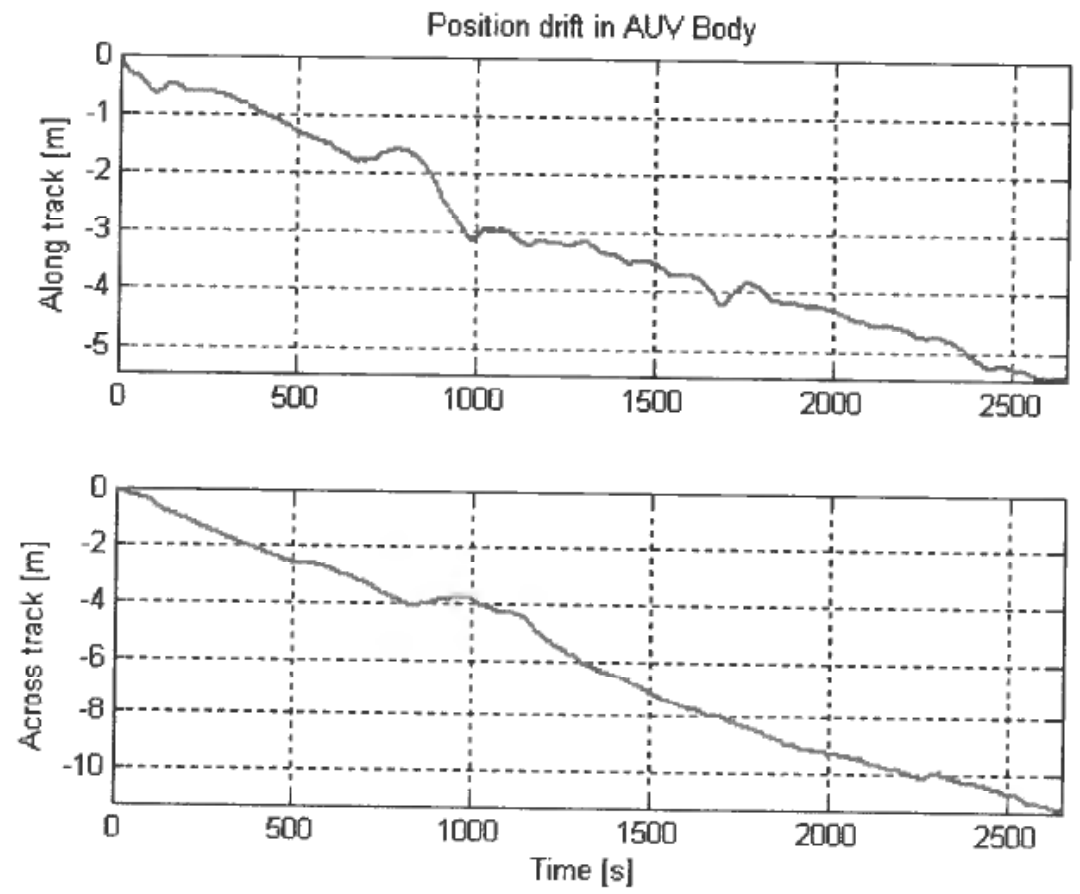

Figure 5. DVL aided INS position drift. 

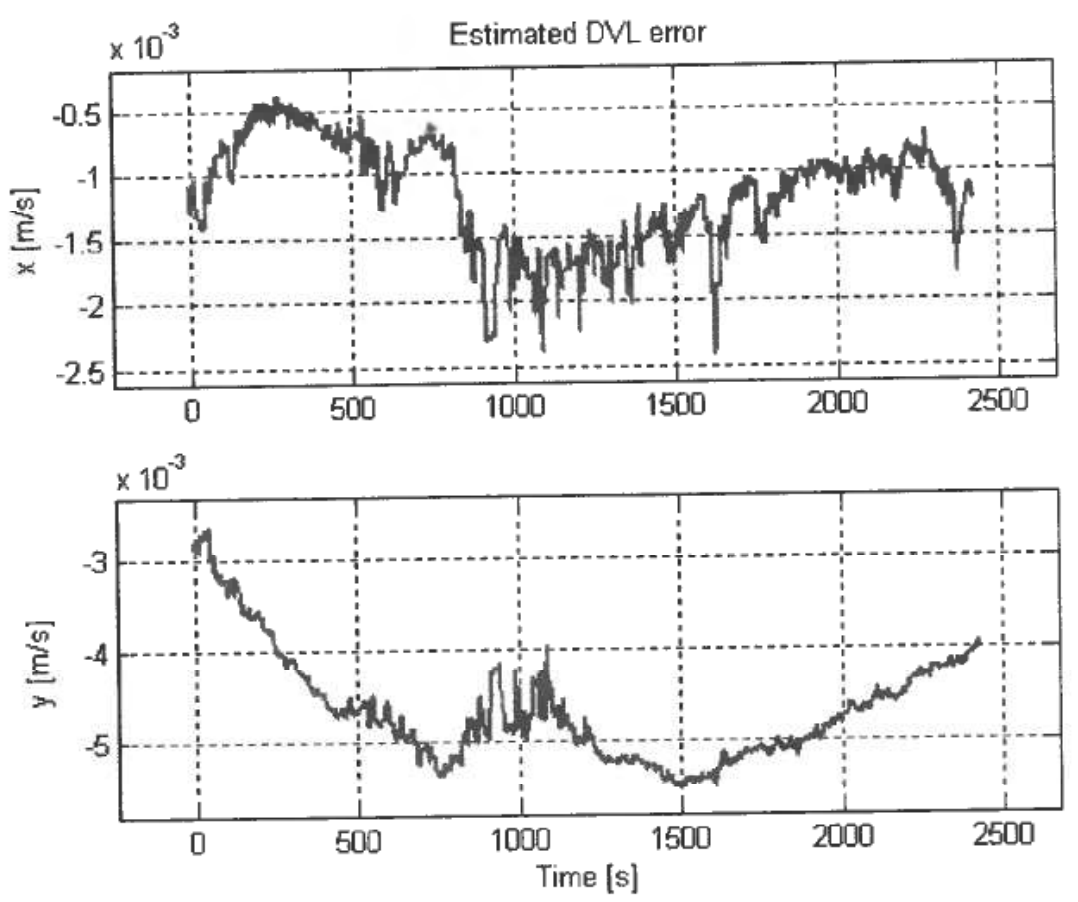

Figure 6. Post-processed DVL error estimate of the same run as shown in Figures 4 and 5. The post-processing was done with DGPS-USBL position updates to improve DVL error observability. The DVL measures velocity in three dimensions, but error estimates are only shown in body $x$ and $y$ for clarity.

aiding only, the position estimate drifts slowly off as explained in Section 3.2. The drift is hardly visible in Figure 4, but is clearly shown in Figure 5, which plots the difference between the DVL aided INS position estimate and the independent position reference. The drift is shown relative to the AUV body (B) system.

Along track error drift is in the order of $4.5 \mathrm{~m}$. Theoretically the WHN-600 $0.2 \%$ scale factor error (refer to Table 2) should contribute with $11 \mathrm{~m}(1 \sigma)$. The good result indicates that the WHN-600 performed better than specification. This is confirmed in Figure 6 where the DVL error has been estimated in the post-processed NavLab run with position measurements. The post-processed Kalman filter estimated the DVL scale factor error to be less than $0.1 \%(<2 \mathrm{~mm} / \mathrm{s}$ at $2 \mathrm{~m} / \mathrm{s}$ AUV speed).

Across track error drift is approximately $11 \mathrm{~m}$. HUGIN 1000 was equipped with an IMU with a gyro bias specification of $0.01 \%$ h (refer to Table 1). According to Equation (1) and Section 3.2, this corresponds to an across track drift of $7.3 \mathrm{~m}(1 \sigma)$ at $60^{\circ}$ latitude. There was virtually no current, and hence marginal sideways speed. The DVL across track error contribution is thus left with the constant y-velocity specification of $1 \mathrm{~mm} / \mathrm{s}$ (Table 2), which contributes with $2.7 \mathrm{~m}(1 \sigma)$. The importance of DVL misalignment estimation was discussed in Section 4. In this run no misalignment of the DVL relative to the IMU was compensated for. Mechanically a production uncertainty of $0.1^{\circ}$ is expected, theoretically contributing to $9.6 \mathrm{~m} \mathrm{drift}$ in this case $(1 \sigma)$. Combining these error sources, the across track error drift is reasonable.

Uncompensated DVL misalignment about the z-axis behaves like a bias in DVL 
$y$-direction when the AUV has a constant forward velocity. This can be seen in the second graph in Figure 6, which shows the estimated DVL bias from the NavLab post-processing with position measurements. The estimated bias in y-direction has an average of about $-4.5 \mathrm{~mm} / \mathrm{s}$, exceeding the constant DVL specification of $1 \mathrm{~mm} /$ $\mathrm{s}$, but corresponding to a misalignment of $0.13^{\circ}$ (neglecting DVL y bias), not far from the expected accuracy of the mechanical mounting. Based on post-processing of a few missions, a DVL misalignment can be estimated. DVL misalignment is an ini-file parameter, enabling the navigation system to compensate for the misalignment in real-time. As mentioned in Section 4, the IMU and DVL are mounted with steering pins, allowing service and inspection without necessitating renewed estimation of DVL misalignment.

\section{Summary}

The Doppler velocity log and the inertial measurement unit are the key AUV navigation sensors enabling submerged operation for long periods of time. To utilize the velocity accuracy offered by the DVL, mounting misalignment between the IMU and the DVL must be minimized, sound speed must be accurately calculated and the sensor data properly time tagged.

HUGIN 1000 sea trials indicate that performance of the RDI WHN-600 is well within specification.

\section{References}

Chance, T. C., Kleiner, A. A. \& Northcutt, J. G. (2000). The HUGIN 3000 AUV, Sea Technology, 41(12), pp. 10-14.

FaLMOITH SCIFNTIFIC (2004). '2' Micro CTD Specification Shect, www.falmouth.com

GADE, K. (2003). NavLab-Overview and User Guide, FFI/Report 2003/02128, Norwegian Defence Research Establishment.

George, R. A., ShuY, J. \& CAUQuil, E. (2003). Deepwater AUV Logs 25,000 Kilometers Under the Sea, Sea Technology, 44(12), pp. 10-15.

Hagen, P. E., Størkersen, N., Vestgård, K. \& Kartvedt, P. (2003). The HUGIN 1000 Autonomous Underwater Vehicle for Military Applications, Proceedings from Oceans
2003, San Diego, CA, USA.

JALVING, B., Bovio, E. \& GADE, K. (2003a) Integrated Inertial Navigation Systems for AUVs for REA Applications, NATO Underwater Research Center Conference Proceedings from MREP 2003, NATO Underwater Research Center May 12-15, La Spezia, Italy.

Jalving, B., Gade, K., Hagen. O. K. \& Vestgård, K. (2003b) A Toolbox of Aiding Techniques for the HUGIN AUV Integrated Inertial Navigation System, Proceedings from Oceans 2003, September 23-25, San Diego, CA, USA.

LESTER, G. (2001). Email to Bjorn Jalving on RDI DVL error budget, 21 June 2001.

RD InSTRUMENTs (1996). Acoustic Doppler Current Profiler, Principles of operation, A practical primer, San Diego, CA, USA.

RD INSTRUMENTs (2003). Workhorse Navigator Doppler Velocity Log (DVL), http://www. dvlnav.com/pdfs/navbro.pdf

VestgaÅrd, K., Hansen, R., Jalving, B. \& Pedersen. O. A. (2001) The HUGIN 3000 Survey AUV, ISOPE-2001, Stavanger, Norway. 